\title{
Micro 3D Culture System using Hyaluronan-Collagen Capsule for Skeletal Muscle-Derived Stem Cells
}

\author{
Kayoko Tono-Okada ${ }^{1,2,5}$, Yoshinori Okada ${ }^{1,3}$, Maki Masuda ${ }^{1,2}$, Akio Hoshi ${ }^{1,4}$, Akira Akatsuka ${ }^{1,3}$, \\ Akira Teramoto ${ }^{5}$, Koji Abe ${ }^{5}$ and Tetsuro Tamaki ${ }^{*}, 1,2$ \\ ${ }^{I}$ Muscle Physiology and Cell Biology Unit; ${ }^{2}$ Department of Regenerative Medicine, Division of Basic Clinical Science; \\ ${ }^{3}$ Teaching \& Research Support Center; ${ }^{4}$ Department of Urology, Division of Surgery, Tokai University School of \\ Medicine, Isehara, Kanagawa, Japan; ${ }^{5}$ Department of Bioscience and Textile Technology, Interdisciplinary Graduate \\ School of Science and Technology, Shinshu University, Ueda, Nagano, Japan
}

\begin{abstract}
In order to hold non-adhesive type cells while maintaining cellular interactions and various autocrine/paracrine factors, a micro 3D culture system using Hyaluronan (HA)-type I collagen capsules was investigated as a possible scaffold for cell transplantation. Skeletal muscle-derived enzymatically extracted cells, which include numerous non-adhesive type stem cells were cultured in conventional liquid DMEM with and without encapsulation in HA-collagen capsules, and cellular proliferation/differentiation were compared. Results indicate that encapsulation does not disturb any cellular proliferation/differentiation after 7 days of culture. Gradual increases in vascular endothelial growth factor are also confirmed in HA-collagen culture, which may be induced by slower diffusion of autocrine/paracrine factors in the capsule and may benefit cellular proliferation/differentiation. Cell-holding capacity of encapsulation was also tested by in vivo transplantation into wide-open muscle scars without fascia. Encapsulation significantly contributes to higher donor cell implantation ratio and damaged muscle mass recovery than that of non-capsulation.
\end{abstract}

Keywords: Encapsulation, non-adhesive cells, scaffold, open injury model, large tissue deficits.

\section{INTRODUCTION}

Among recent efforts to advance clinical therapies, stem cell transplantation is a promising approach to improve tissue reconstitution following traffic accidents and/or large tumor removal. Methods of stem cell transplantation can be broadly divided as follows: 1) transplantation into blood circulation; and 2) direct injection into damaged and/or degenerated local tissues [1-3].

In the case of direct injection into damaged local tissues, it is very important to maintain cellular aggregation of transplanted cells, thus maintaining cell-to-cell relationships, such as autocrine and/or paracrine factors. This process can critically affect the transplanted cellular differentiation capacities in the recipient tissue environment [4]. However, in large tissue deficits, such as those induced in skeletal muscle organs following traffic accidents; it is difficult to maintain cellular aggregation due to the absence of fascia with a wide-open scar area. Thus, biodegradable scaffolds are being developed in order to ensure cellular aggregation.

Sponge or fiber biodegradable scaffolds are well known and are the most commonly applied for this purpose [5-11]. However, it is difficult to maintain non-adhesive stem cells within sponge- or fiber-structure scaffolds, as cells are not held when their size exceeds that of the pores. In contrast, if pore size is much larger than the cells, non-adhesive stem cells may be washed out by tissue fluid.

*Address correspondence to this author at the Muscle Physiology and Cell Biology Unit, Department of Regenerative Medicine, Division of Basic Clinical Science, Tokai University School of Medicine, Shimokasuya-143, Isehara, Kanagawa 259-1193, Japan; Tel: +81-463-93-1121 (ext. 2524); Fax: +81-463-95-0961; E-mail: tamaki@is.icc.u-tokai.ac.jp
In order to address these points, we considered performing three-dimensional (3D) cellular capsule engraftment. For this purpose, capsules need to be produced from biodegradable materials and cells must be stably kept in the capsule until tissue fascicle forms around the damaged area. In addition, encapsulated cells must proliferate and differentiate within these capsules. We decided to use hyaluronan (HA) and type I collagen as materials for biodegradable capsules. We first verified in vitro cellular proliferation and differentiation within the fabricated capsules using skeletal muscle-derived cells, which include adhesive and nonadhesive multipotent stem cells that can give rise to skeletal myogenic, endothelial and adipogenic cells in vitro [12-14].

We also performed in vivo cell transplantation experiments using a skeletal muscle open injury model with and without cell encapsulation, and confirmed cellular implantation capacity.

\section{MATERIALS AND METHODOLOGY}

\section{Materials}

Two types of HA (Bio Sodium Hyaluronate HA9 [MW: $\left.9 \times 10^{5}\right]$ and HA12 [MW: $\left.1.2 \times 10^{6}\right]$ ) were provided by Shiseido Co., Ltd. (Tokyo, Japan) and used for HA-collagen capsule studies. Type I native collagen $(0.5 \% \mathrm{wt} / \mathrm{v})$ derived from bovine dermis (KOKEN, Tokyo, Japan) was used without further modification.

\section{Animals}

Green fluorescent protein transgenic mice (GFP-Tg mice; C57BL/6TgN[act EGFP]Osb Y01) were provided by Dr. M. 
Okabe (Osaka University, Osaka, Japan) [15], and were used for in vitro cell encapsulation and as donor cells for in vivo cell transplantation studies. For cell transplantation, wildtype C57BL/6 mice were used as recipients. Cell encapsulation and/or diffusion were investigated by fluorescence microscopy using a GFP-specific filter (U-MGFPHQ; Olympus, Tokyo, Japan). All experimental procedures were conducted in accordance with the Japanese Physiological Society guidelines for the care and use of laboratory animals, as approved by the Tokai University School of Medicine Committee on Animal Care and Use.

\section{Cell Isolation and Preparation of Freshly Isolated Cell Suspension}

Cell isolation methods are summarized in Fig. (2A). Skeletal muscle-derived cells were extracted from hindlimb muscles (tibialis anterior, extensor digitorum longus, soleus, plantaris, gastrocnemius, and quadriceps femoris) of 4- to 8-week-old GFP-Tg mice, as described previously [12-14, 16-18]. Whole muscles were treated with $0.1 \%$ collagenase type I-A (Sigma-Aldrich, St. Louis, MO, USA) in Dulbecco's modified Eagle's medium (DMEM) containing $7.5 \%$ fetal bovine serum (FBS) with gentle agitation for $2 \mathrm{~h}$ at $37^{\circ} \mathrm{C}$. Skeletal muscle-derived enzymatically extracted cells (Sk-EECs) were filtered through 70-, 40-, and $20-\mu \mathrm{m}$ nylon strainers in order to remove muscle fibers and other debris, followed by washing and resuspension in DMEM containing 20\% FBS (DMEM $/ 20 \%$ FBS). The concentration of freshly isolated cells was adjusted to $3 \times 10^{8}$ cells $/ \mathrm{ml}$ DMEM/20\%FBS (freshly isolated cell suspension, Fig. 2A).

\section{Preparation and Culture of HA-Collagen Capsules Containing Sk-EECs}

Preparation and culture of HA-collagen capsules containing Sk-EECs is outlined in Fig. (2B). First, $100 \mu \mathrm{g}$ of HA9 and $100 \mu \mathrm{g}$ of HA12 powders were mixed and sterilized by autoclave at $121^{\circ} \mathrm{C}$ for $20 \mathrm{~min}$. HA mixtures were then dissolved in $10 \mathrm{ml}$ of DMEM (with $10 \mathrm{mM}$ HEPES), and a $2.0 \%(\mathrm{wt} / \mathrm{v})$ HA9/HA12 mixture solution ( $\mathrm{pH} 7.4$, adjusted by $\mathrm{NaOH}$ ) was obtained. To determine suitable conditions (concentration) for HA-collagen capsules, a graded series of HA9/HA12 solutions $(0.75,1.0$, 1.5 and $2.0 \%$ ) was used. Each HA9/HA12 solution was individually plated ( $5 \mu 1$, plated HA9/HA12 solution in Fig. 2B-a) on culture dishes in quadruplicate using a 96-well tissue multi-plate (total, 16 wells), and each drop was mounted with $10 \mu 1$ of $0.5 \%$ type I native collagen solution, thus producing HA-collagen constructs (Fig. 2B-b). Separately, $1.2 \mu 1$ of freshly isolated cell suspensions were mixed with $3.8 \mu 1$ of each HA9/HA12 solution $(0.75-2 \%$, in quadruplicate) and were slowly injected into plated HA-collagen constructs (Fig. 2B-c). An additional $5 \mu 1$ of $0.5 \%$ native collagen was mounted on the top of each construct and was left to react at room temperature for $15 \mathrm{~min}$ to form HA-collagen capsules (Fig. 2B-d). Capsules were produced in combination with a graded series of HA9/HA12 solutions in order to determine the optimum conditions for HAcollagen capsules.

Produced HA-collagen capsules were washed with DMEM (1 min, $\times 3)$ in order to remove the remaining non-reacted polymers around the capsules. Capsules were cultured in $0.5 \mathrm{ml}$ of DMEM with 20\% FBS containing 100 $\mathrm{mM}$ 2-mercaptoethanol (Wako, Osaka, Japan) without any cytokines. After 2 days of culture, FBS concentration was reduced to $5 \%$, followed by a 5 days of culture. Standard culture was also performed for Sk-EECs without encapsulation as a control.

\section{Skeletal Muscle Open Injury Model and Cell Transplantation}

In order to determine the cell-holding capacity of encapsulation in vivo, we developed a mouse skeletal muscle (tibialis anterior; TA) open injury model and confirmed cellular implantation capacity with and without encapsulation. Schematic drawings of the preparation of the mouse TA muscle open injury model and cell transplantation with and without encapsulation are shown in Fig. (2C). Surgical procedures and cell transplantation were performed under sodium pentobarbital anesthesia $(40 \mathrm{mg} / \mathrm{kg}$, i.p.). The left TA muscle of a wild-type C57BL/6 mouse was exposed by skin incision. A $3-\mathrm{mm}^{2}$ region of the mid portion of TA muscle was then removed to a depth of $2 \mathrm{~mm}$ using ophthalmic scissors, and bleeding was stopped using an electric knife under a dissection microscope. Note that muscle fascia was also removed at this time, leaving a $3 \mathrm{~mm} \times 3 \mathrm{~mm} \times 2 \mathrm{~mm}$ open wound. Removed muscle volume was weighed in order to ensure a similar degree of muscle damage in each mouse (non-encapsulated group, $10.3 \pm 1.0 \mathrm{mg}$; encapsulated group, $11.1 \pm 1.0 \mathrm{mg}$ ). Next, $2 \times 10^{5} \mathrm{GFP}^{+}$cells (Fig. $2 \mathrm{~A}$ ) were transplanted into the open muscle wound with and without encapsulation (see Fig. 2C). Remaining skin was sutured using nylon thread and disinfectant plastic dressing spray (Nobecutane spray; Yoshitomi Chemical, Osaka, Japan). Penicillin-G-potassium (20,000 unit/kg; Meiji-Seika, Tokyo, Japan) was injected subcutaneously in order to prevent infection.

\section{Immunostaining and Histological Analysis}

For immunostaining, capsules were fixed with $4 \%$ paraformaldehyde/0.1 M phosphate buffer (4\% PFA/PB) overnight at $4^{\circ} \mathrm{C}$. Capsules were then washed with a graded sucrose $(5-25 \%) / 0.01 \mathrm{M}$ PBS series and embedded into Tissue-Tek O.C.T. Compound (Miles, Elkhart, IN, USA) and frozen by the metal contact method at $-80^{\circ} \mathrm{C}$. Serial $7-\mu \mathrm{m}$ sections ( $>40$ sections per sample) were obtained. For analysis of capsule formation and composition, 10 sections were stained with Azan (staining for collagen fibrils) and Alcian blue (staining for acid mucopolysaccharides). To confirm cellular proliferative capacity, proliferating cells were stained with mouse monoclonal anti-proliferating cell nuclear antigen (PCNA) antibody (1:1000; Sigma-Aldrich). Mouse anti-MyoD1 monoclonal antibody (1:50; DAKO, Carpinteria, CA, USA), mouse anti-myogenin monoclonal antibody $(1: 75 ;$ DAKO) and rabbit anti-skeletal muscle actin polyclonal antibody (1:200; Abcam, Cambridge, UK) were used to determine skeletal myogenic differentiation. Differentiation into endothelial cell lineages was determined using rat anti-CD31 monoclonal antibody $(1: 100$; BD Pharmingen, San Jose, CA, USA). Cells containing lipid droplets (fat cells) were detected by oil-red $\mathrm{O}$ staining. Reactions were visualized by Alexa Fluor-594 conjugated goat anti-rabbit (1:500; Molecular Probes, Eugene, OR, USA) and goat 
anti-rat secondary antibodies (1:500; Molecular Probes). Nuclei were stained with DAPI. Quantitative analyses were also performed for all immunohistochemical staining. Mean values from five sections were used and mean data from 5 photographs were obtained for each section. Positive cells in each photograph were counted and evaluated as a percentage of total cells/definite area, and mean values were used.

\section{Western Blot Analysis}

In order to confirm whether autocrine and/or paracrine factors were concentrated in the capsule, we selected vascular endothelial growth factor (VEGF) as an indicator. VEGF is reported to be associated with the formation of serial alignments in CD31-positive cells [19, 20], and Sk-EECs include myo-endothelial progenitor cells [12, 13]. Concentrations of VEGF were determined by Western blot analysis.

Samples were obtained before and after 3 and 7 days of culture. At each time point, three capsules were selected and transferred to micro centrifuge tubes. First, capsules were treated with $0.1 \%$ collagenase type I-A solution, followed by incubation for $10 \mathrm{~min}$ at $37^{\circ} \mathrm{C}$ in order to degrade the HA-collagen gel. Subsequently, samples were centrifuged at $11,000 \mathrm{rpm}$ for $15 \mathrm{~min}$ at $4^{\circ} \mathrm{C}$ and the supernatant was transferred to new tubes. Remaining cells were solubilized in lysis buffer containing $10 \mathrm{mM}$ Tris- $\mathrm{HCl}(\mathrm{pH} 7.4), 150 \mathrm{mM}$ $\mathrm{NaCl}, 5 \mathrm{mM}$ EDTA, 1\% TritonX-100, 1\% DOC (deoxycholic acid sodium salt), $0.1 \%$ SDS and protease inhibitor cocktail (1:200; Sigma-Aldrich) on ice for $30 \mathrm{~min}$. Cell lysate solution and previous supernatant were mixed and reacted with an equal volume of $100 \%$ acetone for $60 \mathrm{~min}$ at $-80^{\circ} \mathrm{C}$. After centrifugation at $11,000 \mathrm{rpm}$ for $15 \mathrm{~min}$ at $4{ }^{\circ} \mathrm{C}$, supernatant was removed and the pellet was resolved in Laemmli sample buffer (Bio-Rad, Hercules, CA, USA) containing 5\% 2-mercaptoethanol.

Lysate proteins from three capsules in each group were separated by $12 \%$ SDS-polyacrylamide gel electrophoresis, and were then transferred to a polyvinylidene fluoride (PVDF) membrane (Amersham Biosciences, Piscataway, NJ, USA) at $60 \mathrm{~V}$ for $150 \mathrm{~min}$. After blocking the membrane with $5 \%$ nonfat dried milk for $1 \mathrm{~h}$, the membrane was probed with rabbit anti-VEGF polyclonal antibody (1:200; Santa Cruz Biotechnology, Delaware, CA, USA) at $4^{\circ} \mathrm{C}$ overnight. The membrane was washed with $0.01 \mathrm{M}$ phosphate buffered saline $(0.01 \mathrm{M} \mathrm{PBS}) / 0.1 \%$ Triton X-100 (PBS-T), and was then incubated in the peroxidase-linked anti-rabbit $\operatorname{IgG}$ antibody for $60 \mathrm{~min}$ at room temperature. Detection was performed using an ECL plus Western blotting detection system (Amersham Biosciences). The intensity of the bands was determined using a Light Capture chemiluminescent imaging system (ATTO, Tokyo, Japan).

\section{Histological Analysis after in vivo Cell Transplantation}

Histological analysis was performed at 4 weeks after transplantation. Animals were overdosed with sodium pentobarbital $(60 \mathrm{mg} / \mathrm{kg}$, i.p.). First, the implanted region was observed by fluorescence dissection microscopy and in situ cellular implantation ratio was evaluated as the percentage of $\mathrm{GFP}^{+}$area against total TA surface area (see Fig. 9A and B). Animals were then perfused with warm 0.01 M PBS through the left ventricle, followed by fixation with $4 \%$ PFA/PB. TA muscles from both legs were then removed and weighed, and post-fixed overnight with $4 \%$ PFA/PB at $4^{\circ} \mathrm{C}$. Samples were washed with a graded sucrose $(5-25 \%) / 0.01 \mathrm{M}$ PBS series, and quick frozen in isopentane, after which serial $7-\mu \mathrm{m}$ cross-sections were obtained. Five sections/muscle were prepared for further quantitative analysis. Sections were observed by fluorescence light microscopy and cellular implantation ratio was also determined as a percentage of $\mathrm{GFP}^{+}$cellular area against total TA cross sectional area (see Fig. 9D and E). For the area analysis on total TA muscle surface, whole cross sections and implanted $\mathrm{GFP}^{+}$cells and/or tissues was performed using software Stereo Investigator (Mbf BIOSCIENCE, Williston, VT, USA). Sections were also stained with monoclonal $\mathrm{Cy} 3$ conjugated anti-smooth muscle actin ( $\alpha$-SMA, Sigma-Aldrich) in order to detect blood vessels.

\section{Statistical Analysis}

Data are expressed as means \pm SD. Differences between the non-encapsulated and encapsulated groups were determined using Student's $t$-test. Differences were considered to be statistically significant at $\mathrm{P} \leq 0.05$.

\section{RESULTS}

\section{Representative Adhesive and Non-Adhesive Cells in Skeletal Muscle}

As we have reported, skeletal muscle includes numerous non-adhesive cells, as well as adhesive cells $[12,13]$. When

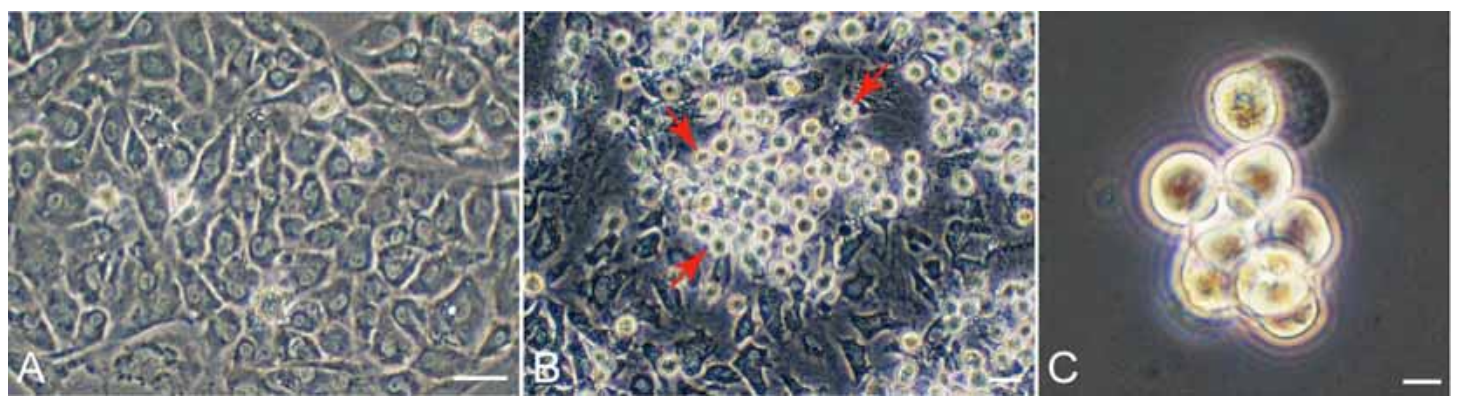

Fig. (1). Representative adhesive (A) and non-adhesive (B: red arrows, C) stem cells derived from skeletal muscle cultured for 5 days in conventional 2D (liquid) culture. Typical adhesive cells showing cobblestone-like formation (A), and non-adhesive cells distributed above the adhesive cell layer (B: red arrows). Non-adhesive cells were round in shape and formed aggregations (sphere-like colonies, $\mathbf{C})$. Scale bar in $\mathbf{A}$ and $\mathbf{B}=50 \mu \mathrm{m}$; in $\mathbf{C}=10 \mu \mathrm{m}$. 
Sk-EECs were culture in a conventional 2D liquid culture system for 5 days, adhesive cells showed cobblestone-like
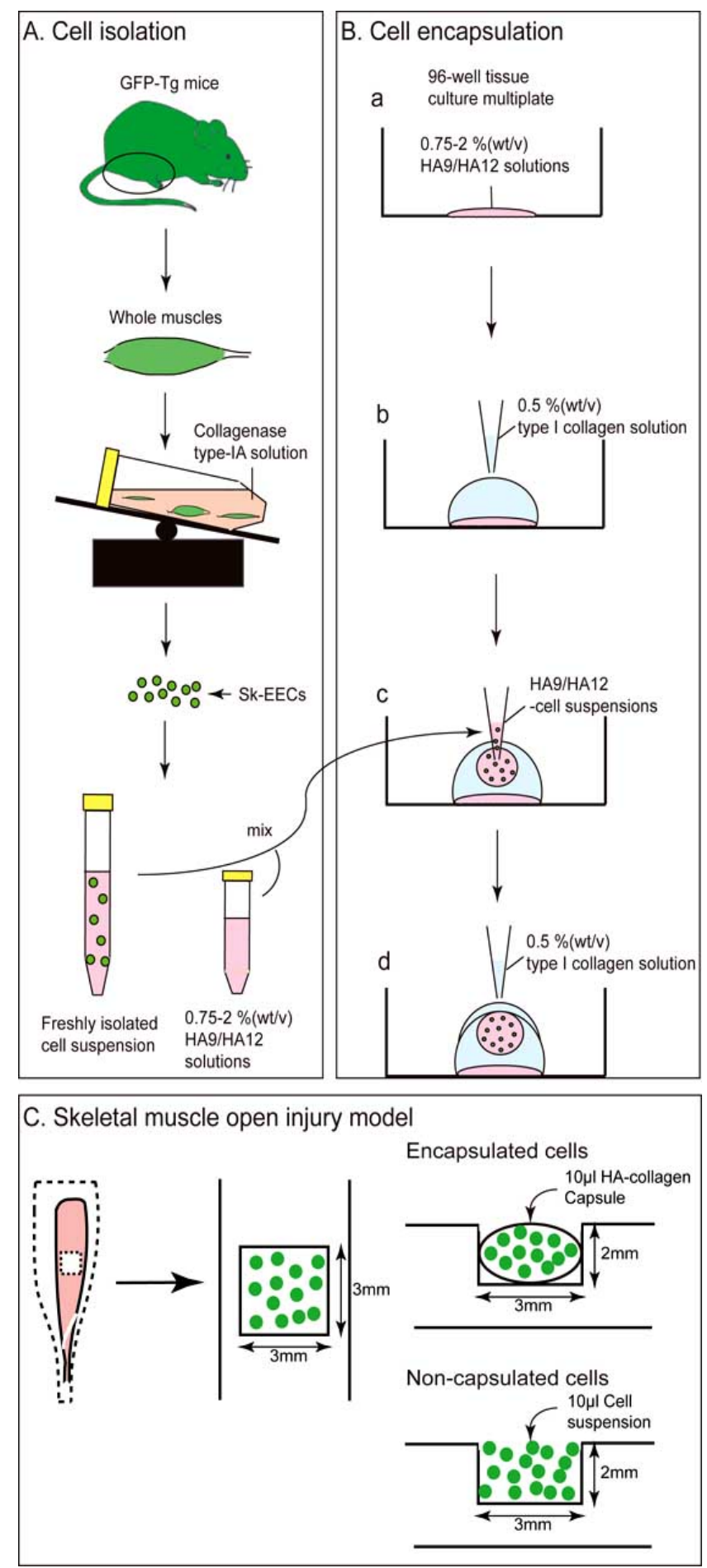

Fig. (2). Schematic description for the preparation of skeletal muscle-derived enzymatically extracted cell (Sk-EEC) suspension (A), encapsulation of Sk-EECs into HA-collagen capsules (B) and preparation of skeletal muscle open injury model (C). Optimal conditions (concentration) of HA-collagen capsules for the complete encapsulation of Sk-EECs were determined using a graded series of HA9/HA12 solutions $(0.75,1.0,1.5$ and $2.0 \%)$ and $0.5 \%(\mathrm{wt} / \mathrm{v})$ type I collagen solution $(\mathbf{B a}-\mathrm{d})$. formation, as typically observed for endothelial cell culture (Fig. 1A). On the other hand, non-adhesive cells were distributed above the adhesive cell layer (Fig. 1B: red arrows). These non-adhesive cells were round in shape and could proliferate and form cell aggregates, such as sphere-like colonies, even in a floating state (Fig. 1C), and multipotent stem cells were present in these non-adhesive cell populations [12, 13]. For this reason, we considered encapsulation of these cells in order to maintain cell-to-cell relationships, even in large tissue defects after direct cell transplantation.

\section{Sk-EEC Encapsulation in HA-Collagen Capsules}

In order to determine the optimal concentration of HA9/ HA12 solutions, HA-collagen capsules containing Sk-EECs were produced in combination with a graded series of HA9/HA12 solutions using both plates and cell suspensions. Capsules were cultured for 1 week. We then found that the optimal concentration of HA9/HA12 solutions was $2 \%$ on the plates and $0.75 \%$ in the cell suspensions. Under these conditions, there was no leakage of cells from the capsules (Fig. 3A). In contrast, when capsules were made with $\leq 2 \%$ HA9/HA12 solutions on the plates and $\geq 0.75 \%$ in the cell suspensions, most capsules shrunk and allowed cell diffusion during culture (Fig. 3B-C). Under optimal conditions, capsules began to change their shape to disk-like structures of approximately $4 \mathrm{~mm}$ in diameter and $1 \mathrm{~mm}$ in thickness within the first hour of cell culture (Fig. 3D). Each capsule included approximately $3.5 \times 10^{4}$ cells.

\section{Macroscopic Observation of HA-Collagen Capsules Containing Sk-EECs}

A macroscopic view (under dissection microscope) of HA-collagen capsules containing Sk-EECs after 7 days of culture is shown in Fig. (4A). The HA-cell suspension layer (white arrowhead in Fig. 4A) was surrounded by a collagen layer (red arrows in Fig. 4A). This collagen layer formed a gel due to its relationship with HA; thus, a HA-collagen matrix formed, and was surrounded by the cell layer (outside edge of the capsule). Several tube formations, probably myotubes, were observed in the cell layer (arrowheads in Fig. 4B). Numerous myotube-like formations can be seen more clearly at higher magnifications (arrowheads in Fig. 4C). These features are quite similar to those in normal control culture of non-encapsulated Sk-EECs (arrowheads in Fig. 4D).

\section{Histological Analysis of Capsules and Immunostaining for Containing Sk-EECs}

Capsule composition was confirmed by Azan-Alcian blue staining (collagen fibril and acid mucopolysaccharide staining). Uniform Azan-Alcian blue staining was seen in the HA-collagen matrix gel layer without any cells (Fig. 5A and B). This confirmed that cells were stably kept in the cell suspension layer. On immunohistochemical analysis, a large number of anti-PCNA-positive cells were detected throughout the HA-cell suspension layer (pink reactions in Fig. 6A-a). Similarly, MyoD- (Fig. 6A-b) and myogenin(Fig. 6A-c) positive cells, which were considered to be skeletal myogenic cells, can be seen in the HA-cell suspension layer (pink reactions). Several cells positive for 

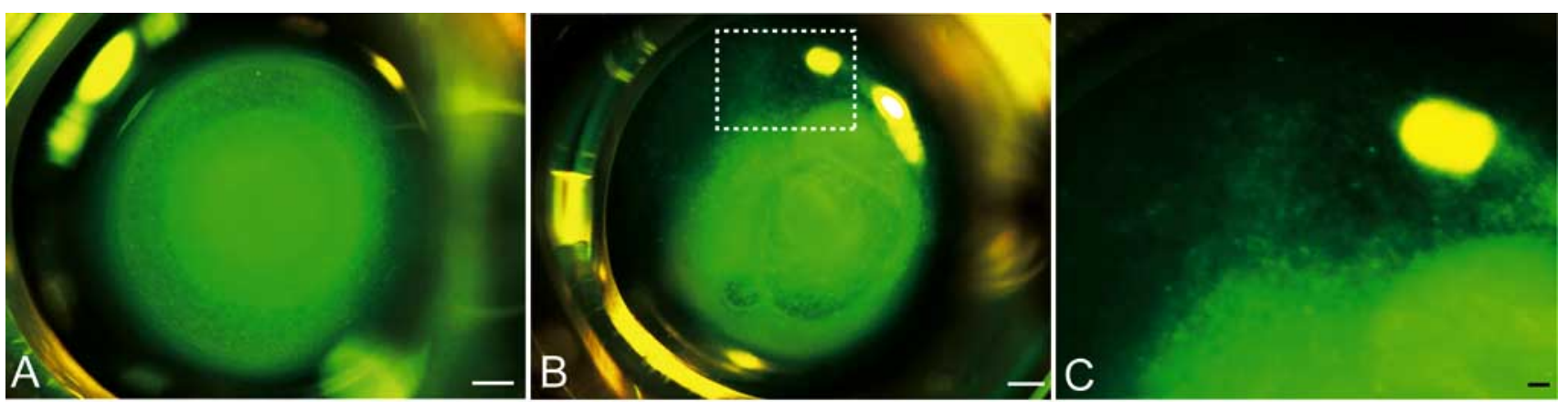

Approx. $4 \mathrm{~mm}$

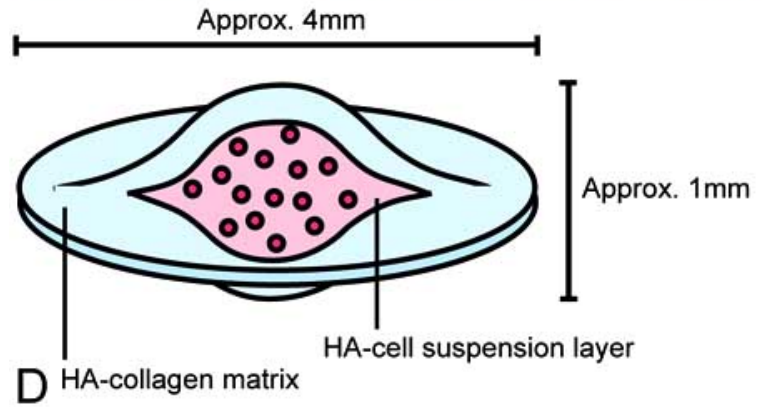

Fig. (3). Macroscopic observation of complete (A) and incomplete (B and C) encapsulation of Sk-EECs by fluorescence dissection microscopy and schematic description of HA-collagen capsule (D). Dotted frame in B corresponds to panel C. The HA-cell suspension layer was completely surrounded by HA-collagen matrix (A). The capsule wall (HA-collagen matrix) was partially broken and inner cells diffused to the outside edge of the capsule (B and $\mathbf{C})$. High magnification photograph clearly shows diffused GFP ${ }^{+}$cells $(\mathbf{C})$. Capsules had a disk-like shape of approximately $4 \mathrm{~mm}$ in diameter and $1 \mathrm{~mm}$ in thickness (D). Scale bar in $\mathbf{A}$ and $\mathbf{B}=500 \mu \mathrm{m}$; in $\mathbf{C}=100 \mu \mathrm{m}$.
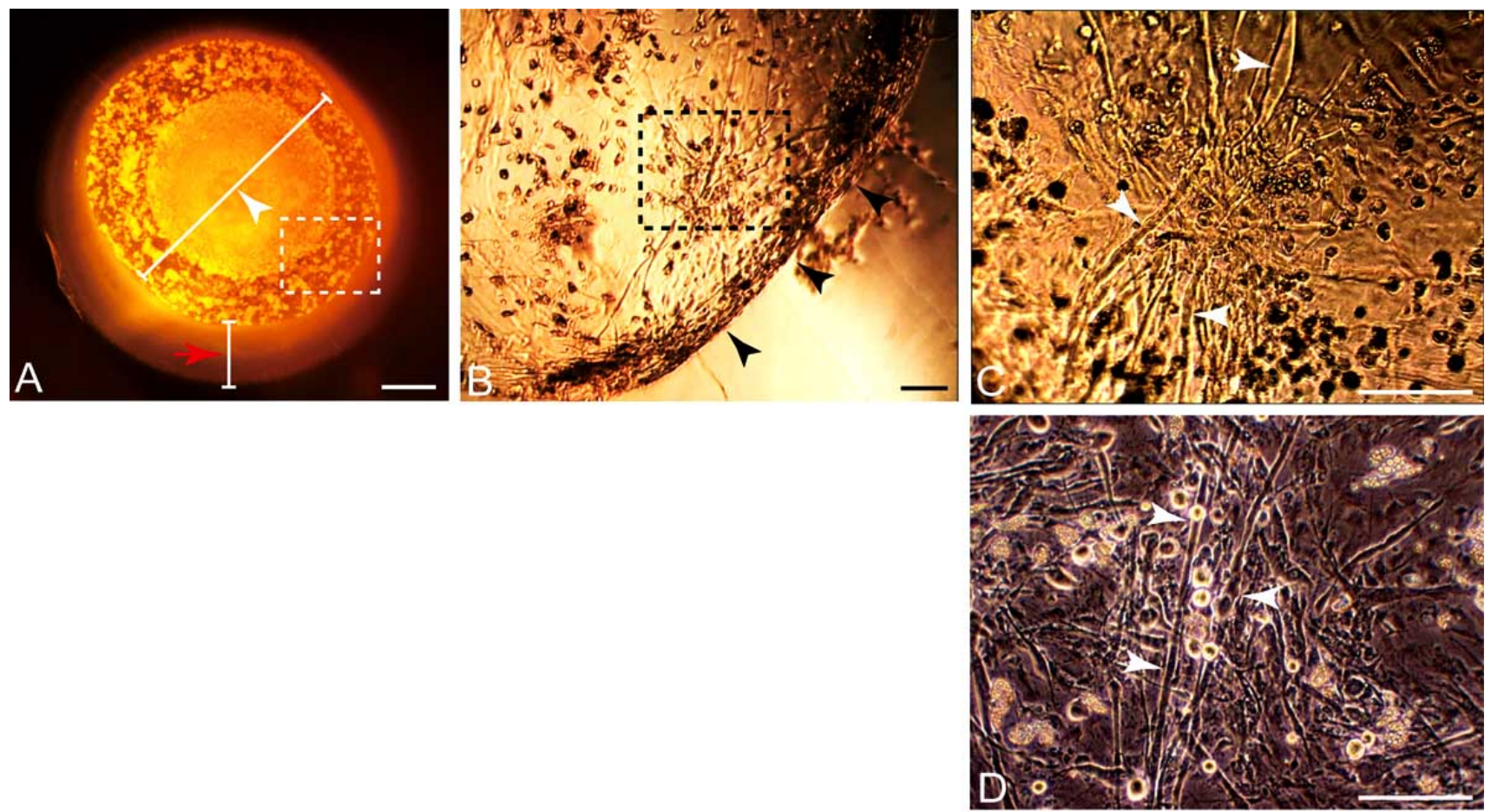

Fig. (4). Macroscopic view of HA-collagen capsule containing Sk-EECs under dissection microscope (A) and phase-contrast inverted light micrographs of cultured Sk-EECs (B and C). Dotted frame in A corresponds to panel B, and dotted square in B corresponds to panel C. Panel A shows typical macroscopic observations of HA-collagen capsule after 7 days of culture. HA-cell suspension layer (A, white arrowhead) and HA-collagen matrix (A, red arrow) can be seen surrounding the capsule. Several tube formations were observed at the border of the cell layer (B, arrowheads). Abundant myotube-like formations can be seen at higher magnifications (C, arrowheads). Similar myotube-like formations can also be observed in normal Sk-EEC culture (D, arrowheads). Scale bar in $\mathbf{A}=500 \mu \mathrm{m}$; in $\mathbf{B}$ and $\mathbf{C}=100 \mu \mathrm{m}$. 


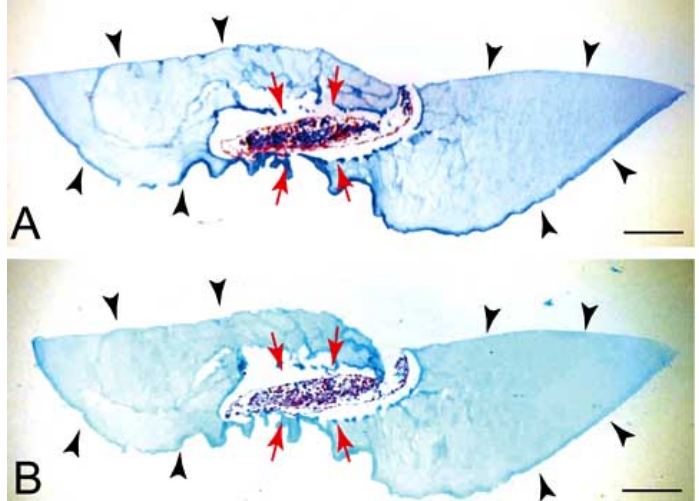

Fig. (5). Histological sections of HA-collagen capsule containing Sk-EECs after 7 days of culture stained with Azan (A; marker of collagen fibrils) and Alcian blue (B; marker of acid mucopolysaccharides). Uniform staining of Azan and Alcian blue can be seen in HA-collagen matrix layers (blue reactions, black arrowheads in $\mathbf{A}$ and $\mathbf{B})$. In addition, cells remained stable in the cell suspension layer (red reactions, red arrows in $\mathbf{A}$ and $\mathbf{B}$ ). In this photomicrograph, note that samples were affected by fixation in $4 \%$ $\mathrm{PFA} / \mathrm{PB}$; thus, HA-collagen matrix reduced in size and Sk-EECs were disrupted. Scale bars $=100 \mu \mathrm{m}$. anti-skeletal muscle actin was evident in the capsule (Fig. 6A-d) and some of those were mono nucleated (arrows in Fig. 6A-e), but multi-nucleated tubes were also positive for anti-skeletal muscle actin as were the myotube (arrowheads Fig. 6A-e), thus further confirming differentiation into skeletal muscle cell lineage. Serial alignments of CD31-positive cells were also located near the border between the HA-cell suspension layer and the HA-collagen matrix (arrowheads in Fig. 6A-f and -g). Cells showing oil droplet-like staining for oil red $\mathrm{O}$, typical of fat cells, were also detected in the HA-cell suspension layer (red reactions in Fig. 6A-h and -i). Taken together, these results indicate that cells in the HA-cell suspension layer proliferated $\left(\mathrm{PCNA}^{+}\right)$and differentiated into skeletal myogenic (MyoD and myogenin ${ }^{+}$), vasculogenic $\left(\mathrm{CD} 31^{+}\right)$and fat cells (Oil red $\mathrm{O}^{+}$) during cell culture.

Similar trends were consistently observed in conventional, normal cell cultures of non-encapsulated Sk-EECs (Fig. 6B, a; PCNA, b; MyoD, c; myogenin, d; SkMA, e; CD31, f; oil red O). However, note that serial alignment of CD31-positive cells, such as seen in the HA-collagen capsules, was not seen in conventional cell culture of Sk-EECs.
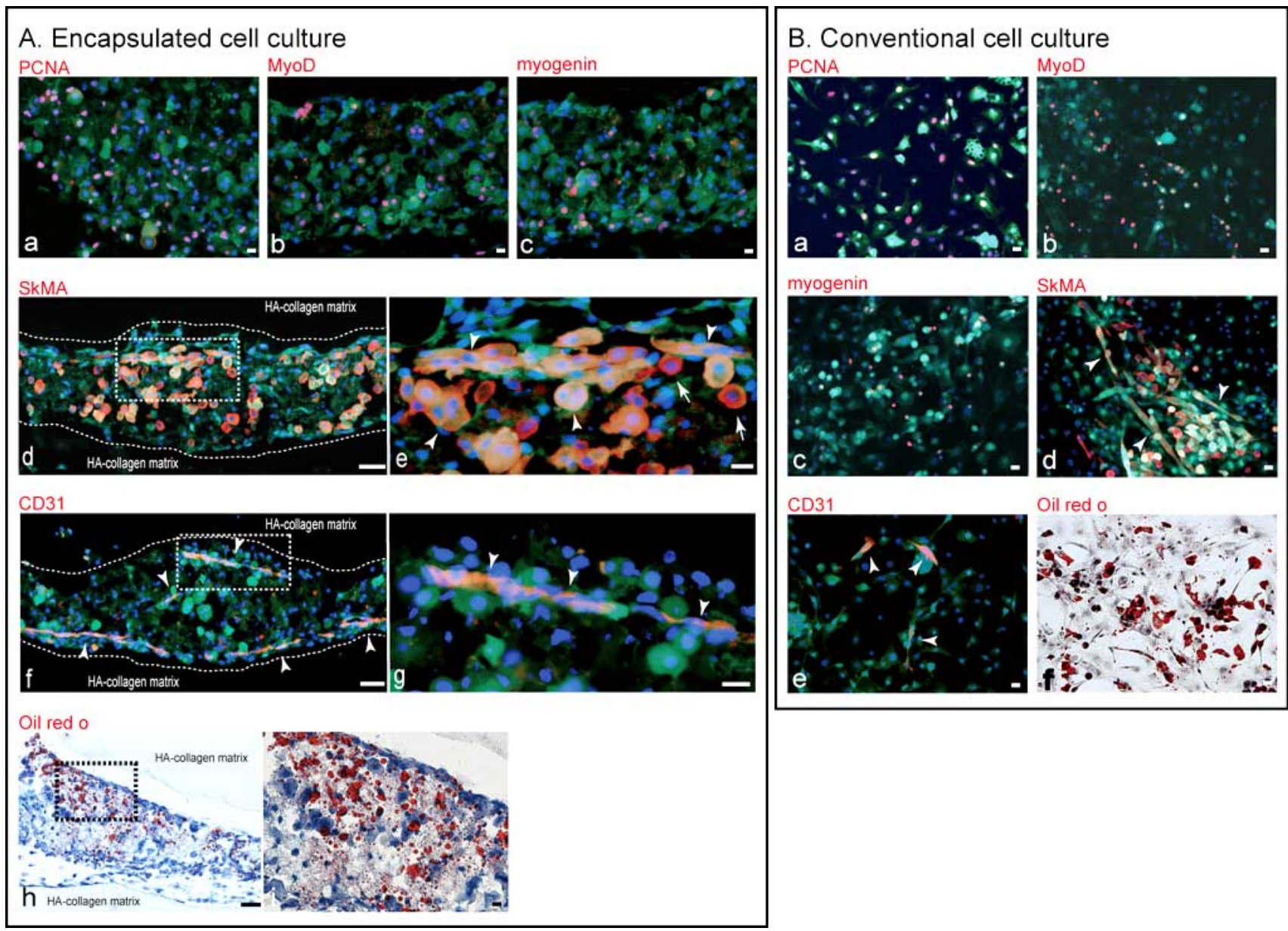

Fig. (6). Immunohistochemical detection of proliferating, myogenic, vascular (endothelial and smooth muscle cells) and adipocytes after 7 days in the HA-collagen capsule culture (A) and conventional normal cell culture (B). Panel A-e shows a higher magnification view of the corresponding area in A-d (dotted frame). Similarly, panel A-g shows a higher magnification view of the dotted frame in A-f. The same trends were seen in both culture systems, suggesting that HA-collagen encapsulation did not disturb cellular growth in culture. Scale bars in $\mathbf{A}-\mathbf{C}, \mathbf{E}, \mathbf{G}, \mathbf{I}$ and $\mathbf{J}-\mathbf{O}=10 \mu \mathrm{m}$; in $\mathbf{D}, \mathbf{F}$ and $\mathbf{H}=50 \mu \mathrm{m}$. 
Quantitative analyses of immunohistochemical staining are shown in Fig. (7). The ratios of proliferative $\left(\mathrm{PCNA}^{+}\right)$, myogenic $\left(\mathrm{MyoD}^{+}\right)$and myogenic differentiated (myogenin ${ }^{+}$ and $\mathrm{SkMA}^{+}$) cells were similar in both culture systems.

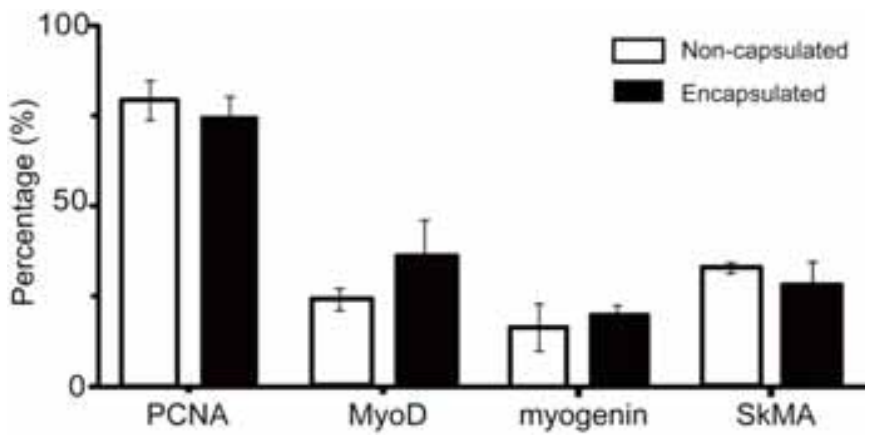

Fig. (7). Quantitative analyses of immunohistochemical staining. Values are expressed as percentages based on the following equation: (number of positive cells/total cells) $\times 100$. There were no significant differences in the 4 immunohistochemical analyses $(\mathrm{P}>0.05)$.

\section{Concentration of VEGF Protein in HA-Collagen Capsules}

Expression of VEGF was detected by Western blot analysis of Sk-EEC-containing HA-collagen capsules (Fig. 8). Expression bands of the expected size (approximately 21 $\mathrm{kDa}$ ) were apparently stronger as culture duration increased, thus confirming a gradual increase in VEGF protein levels within the capsule.

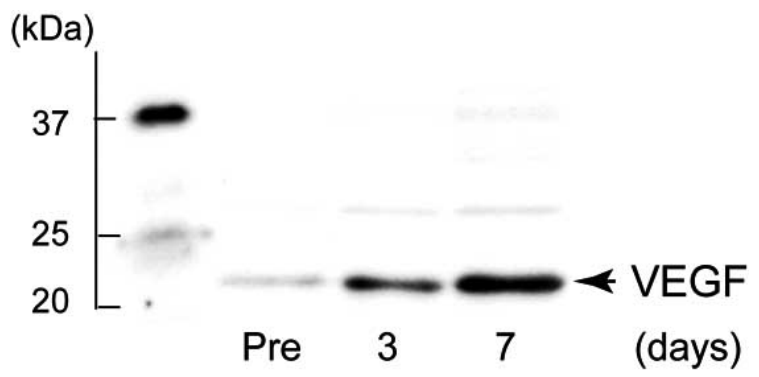

Fig. (8). Western blot analysis of VEGF in encapsulated Sk-EECs before and after 3 and 7 days of culture. Gradual increases in VEGF protein concentrations can be seen with culture duration. Pre; before culture.

Cell-Holding Capacity of Encapsulation after Direct Transplantation into Wide Open Muscle Wound in vivo

In vivo cell-holding capacity of encapsulation following cell transplantation was investigated using an open skeletal muscle injury model. Final body and muscle mass, and mass recovery ratio of transplanted muscle are summarized in Table 1. Body and control TA muscle mass did not significantly differ between the two groups; however, treated TA muscle mass and recovery ratio were significantly higher in the encapsulated group.

Histological detection of cellular implantation and these quantitative data are presented in Fig. (9). Under fluorescence dissection microscopy, an apparently large number of $\mathrm{GFP}^{+}$tissues were seen after encapsulated cell transplan- tation (B) when compared to non-encapsulated cell transplantation (A). When green $\left(\mathrm{GFP}^{+}\right)$tissue surface areas were

Table 1. Final Body and Muscle Mass, and Mass Recovery Ratio of Transplanted Muscle

\begin{tabular}{|c|c|c|}
\hline Experimental group & Non-capsulated $(\mathbf{n}=\mathbf{4})$ & Encapsulated $(\mathbf{n}=6)$ \\
\hline \hline Body mass $(\mathrm{g})$ & $27.5 \pm 7.2$ & $34.8 \pm 7.1$ \\
\hline TA muscle mass $(\mathrm{mg})$ & & $55.6 \pm 7.1$ \\
Control side & $49.3 \pm 5.7$ & $46.2 \pm 7.2 *$ \\
Operated side & $35.4 \pm 4.2$ & $78.0 \pm 3.4 *$ \\
\hline Recovery ratio $(\%)$ & $71.8 \pm 2.2$ & \\
\hline
\end{tabular}

evaluated as a percentage against total TA muscle surface area, significantly (about 2.5 hold) higher values were observed in the encapsulated cell transplantation group (C). In addition, in the histological muscle cross-sections obtained from the portions indicated by white lines in panels A and B, encapsulated transplantation muscle (E) also showed an apparently large number of $\mathrm{GFP}^{+}$cells when compared to non-encapsulated transplantation muscle (D), suggesting that transplanted donor cells survive well in both surface and deep areas of damaged muscle. Mean percentage of $\mathrm{GFP}^{+}$ area on histological sections was also significantly (about 4 fold) higher in the encapsulated group (F). Typical immunohistochemical detection of blood vessels in the encapsulated transplantation muscle is shown in Fig. (9G and $\mathbf{H}$ ). Several blood vessels positive for anti- $\alpha \mathrm{SMA}$ were formed by donor-derived $\mathrm{GFP}^{+}$cells (arrows in Fig. 9G and $\mathbf{H}$ ) located among $\mathrm{GFP}^{+}$donor-derived muscle fibers. Interestingly, active blood vessel formation was observed throughout the encapsulated transplantation muscles.

\section{DISCUSSION}

In the present study, we found that HA-collagen capsules were able to hold isolated adult skeletal muscle-derived cells, including a large number of non-adhesive cells, and that encapsulation did not disrupt cellular proliferation or differentiation of these cells in vitro. In addition, encapsulation also contributed to maintaining the concentration of autocrine/paracrine factors within the capsule. These results clearly indicate that HA-collagen based capsules are useful as scaffolds for non-adhesive-type stem cells. Such capsules will likely benefit direct stem cell transplantation into local tissues.

In the case of stem cell transplantation into blood circulation, such as hematopoietic stem cells [21] and bone marrow-derived stem cells [22], scaffolds are not necessary. However, in the case of direct injection of stem cells into damaged and/or degenerated local tissues with wide open areas in the absence of fascia, maintaining cell-to-cell relationships, such as autocrine and/or paracrine factors, is very important. Such relationships are particularly important in first 1-2 weeks after transplantation [16, 23], as stem cells may affect one another with regard to proliferation and differentiation during this period. Our HA-collagen capsules maintained their structure for more 2 weeks of culture (data 

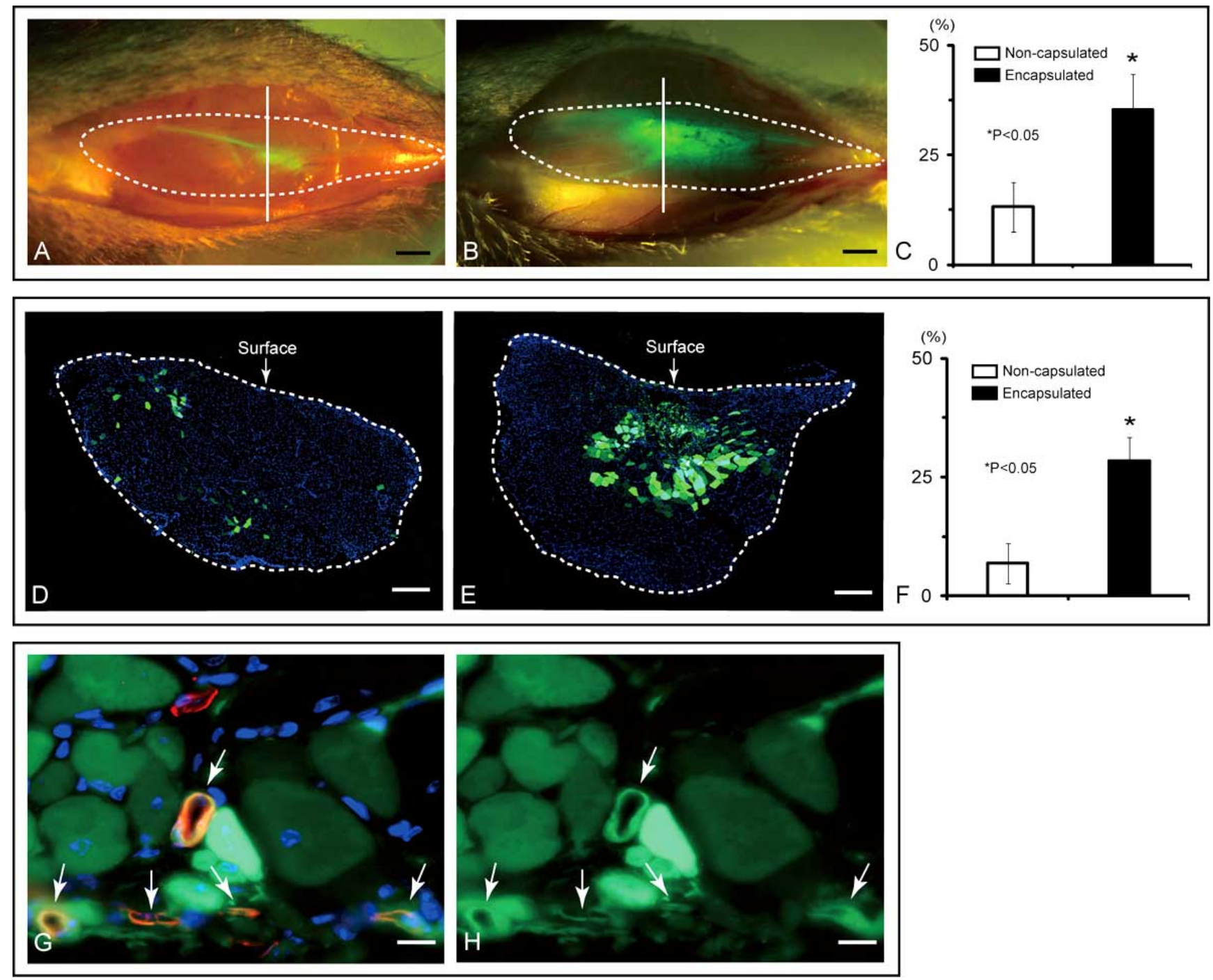

Fig. (9). Typical detection of $\mathrm{GFP}^{+}$donor-derived cells after direct cell transplantation with and without encapsulation into a wide open muscle wound. Large volumes of $\mathrm{GFP}^{+}$green tissues can be seen in encapsulated transplantation muscle in whole TA muscle in situ (dotted lines in $\mathbf{A}$ and $\mathbf{B}$ ) and in cross sections (dotted lines in $\mathbf{D}$ and $\mathbf{E}$ ). Panels $\mathbf{A}, \mathbf{D}$ : non-encapsulated; Panels $\mathbf{B}, \mathbf{E}, \mathbf{G}, \mathbf{H}$ : encapsulated. White lines in panels $\mathbf{A}$ and $\mathbf{B}$ correspond to the cross sections shown in panels D and E. Panels $\mathrm{C}$ and $\mathrm{F}$ show the results of quantitative analysis based on the photographs such as in $\mathbf{A}$ and $\mathbf{B}$, and $\mathbf{D}$ and $\mathbf{E}$. Values (\%) were obtained by following equation; $\mathbf{C}$ : (GFP ${ }^{+}$green muscle surface area/total TA surface area) $\times 100, \mathbf{F}:\left(\mathrm{GFP}^{+}\right.$cell area/total cross-sectional area) $\times 100$. Panels $\mathbf{G}$ and $\mathbf{H}$ show the detection of donor-derived $\mathrm{GFP}^{+}$blood vessels using anti- $\alpha \mathrm{SMA}$ (arrows). Bars in $\mathbf{A}$ and $\mathbf{B}=150 \mu \mathrm{m}, \mathbf{D}$ and $\mathbf{E}=200 \mu \mathrm{m}$, and $\mathbf{G}$ and $\mathbf{H}=20 \mu \mathrm{m}$.

not shown) and allow proliferation of cells. It is generally considered that transplanted exogenous materials are surrounded by thin fascia within 2 weeks in vivo, and thus cells do not need to be held for any longer. Therefore, the next step of our experiment was to adjustment the composition of these capsules to dissolve after around 2 weeks after transplantation in vivo and to confirm whether cell encapsulation contributes to cell-holding after direct transplantation into damaged and/or degenerated local tissues in the absence of fascia.

As expected, encapsulation significantly contributed to cell-holding after direct cell transplantation using the mouse open muscle injury model and large volumes of donorderived $\mathrm{GFP}^{+}$cells/tissues were observed in the encapsulated transplantation group (Fig. 9A-F). In contrast, few $\mathrm{GFP}^{+}$ cells were detected in the non-encapsulated transplantation group. It is conceivable that cells transplanted into wide open areas defused soon after transplantation, thus resulting in fewer implanted donor cells in the non-encapsulated group (Fig. 9A, C, D, F). In addition, the better preservation of donor cells in the damaged area is probably reflected by the significantly better recovery of muscle mass in the encapsulated transplantation group.

Interestingly, serial alignments of CD31-positive cells were observed in the present study (Fig. 6A-f and g) in the absence of additional exogenous growth factors. This trend was not seen in normal Sk-EEC culture or in previous muscle-derived stem cell cultures $[12,13]$. This may be because the concentration of VEGF increased with time in the capsule. The capsule wall, which consisted of HA and collagen hydrogel, promoted slower diffusion of VEGF to the outside of the capsule, and temporarily increased VEGF 
levels may contribute to the formation of serial alignments of CD31-positive cells. Serial alignments of CD31 cells are a typical feature of endothelial cell cultures treated with angiogenic growth factors such as VEGF and basic fibroblast growth factor (bFGF) [22, 23].

We previously showed that skeletal muscle-derived myo-endothelial progenitor cells $\left(\mathrm{CD} 34^{+} / 45^{-}\right.$; Sk-34 cells) had non-adhesive and adhesive properties in vitro, and adhesive cells can give rise to endothelial cells positive for DiI-Ac-LDL (1,1'-dioctadecyl-3,3,3',3'-tetramethylindocarbocyanine perchlorate) and Isolectin $\mathrm{B}_{4}$ (Griffonia Simplicifolia Lectin I), which are endothelial cell markers [12, 13]. The present Sk-EECs were isolated by the same methods as for Sk-34 cells [12-14, 16-18], and they were not sorted (total cell fraction); thus, they included Sk-34 and other stem cells $[13,14,16,17]$. Skeletal muscle also includes fibroblasts in their connective networks [24, 25], which may have contaminated the Sk-EECs and contributed to bFGF expression. For these reasons, concentrated VEGF and bFGF probably induced serial alignment of skeletal muscle derived myo-endothelial cells. Other growth factors and/or cytokines with higher molecular weights than VEGF may also become concentrated in the capsules. Reports that direct cell-cell interaction between fibroblasts induces expression of VEGF [24, 25] also support the present results. However, encapsulated Sk-EECs showed copacetic proliferation and differentiation, thus further indicating that nutritional and other factors in the culture medium continuously affected Sk-EEC growth in the capsule, which suggests that low-weight molecules can pass through the HA-collagen capsule with relative ease, while larger molecules and/or vigorously expressed molecules accumulated.

Interestingly, the present evidences suggested a trend that the HA-collagen capsules accelerate blood vessel formation by Sk-EECs in vitro (Fig. 6). In fact, the present in vivo transplantation with encapsulation showed vigorous formation of donor-derived blood vessels (Fig. 9G and H) that may have been better than that reported previously [16, 17]. However, the direct relationship among in vivo cell transplantation, blood vessel formation and HA-collagen gel remains unclear and is currently under further investigation.

\section{CONCLUSION}

We found that the present HA-collagen capsule micro 3D culture system had the following benefits: 1) non-adhesive cells were kept within the capsules; 2) encapsulated cells proliferated and differentiated without any disturbance; 3) capsules also contributed to maintaining concentrations of autocrine/paracrine factors; and 4) encapsulation significantly contributed to cell-holding after in vivo cell transplantation into wide open wounds in muscle tissue. Therefore, this method may be useful for direct cell transplantation therapy into local tissues damaged after traffic accidents and/or following the surgical removal of large tumors.

\section{ACKNOWLEDGEMENTS}

This work was supported by a Grant-in-Aid for Scientific Research from the Ministry of Education, Science and Culture of Japan, and the Tokai University Research Aid and
Global Center of Excellence Program by the Ministry of Education, Culture, Sports, Science and Technology.

\section{REFERENCES}

[1] Mitterberger M, Pinggera GM, Marksteiner R, et al. Adult stem cell therapy of female stress urinary incontinence. Eur Urol 2008; 53: 169-75.

[2] Maitra B, Szekely E, Gjini K, et al. Human mesenchymal stem cells support unrelated donor hematopoietic stem cells and suppress T-cell activation. Bone Marrow Transplant 2004; 33: 597-604.

[3] Vulliet PR, Greeley M, Halloran SM, et al. Intra-coronary arterial injection of mesenchymal stromal cells and microinfarction in dogs. Lancet 2004; 363: 783-4.

[4] Tang YL, Zhao Q, Qin X, et al. Paracrine action enhances the effects of autologous mesenchymal stem cell transplantation on vascular regeneration in rat model of myocardial infarction. Ann Thorac Surg 2005; 80: 229-37.

[5] Mueller SM, Shortkroff S, Schneider TO, et al. Meniscus cells seeded in type I and type II collagen-GAG matrices in vitro. Biomaterials 1999; 20: 701-9.

[6] Park SN, Park JC, Kim HO, et al. Characterization of porous collagen/hyaluronic acid scaffold modified by 1-ethyl-3-(3-dime-thylaminopropyl) carbodiimide cross-linking. Biomaterials 2002; 23: 1205-12.

[7] Hemmrich K, von Heimburg D, Rendchen R, et al. Implantation of preadipocyte-loaded hyaluronic acid-based scaffolds into nude mice to evaluate potential for soft tissue engineering. Biomaterials 2005; 26: 7025-37.

[8] Grigolo B, De Franceschi L, Roseti L, et al. Down regulation of degenerative cartilage molecules in chondrocytes grown on a hyaluronan-based scaffold. Biomaterials 2005; 26: 5668-76.

[9] Yang SH, Chen PQ, Chen YF, et al. An in vitro study on regene-ration of human nucleus pulposus by using gelatin/chondroitin-6-sulfate /hyaluronan tri-copolymer scaffold. Artif Organs 2005; 29: 806-14.

[10] Chen G, Sato T, Ohgushi H, et al. Culturing of skin fibroblasts in a thin PLGA-collagen hybrid mesh. Biomaterials 2005; 26: 2559-66.

[11] Bashur CA, Dahlgren LA, Goldstein AS. Effect of fiber diameter and orientation on fibroblast morphology and proliferation on electrospun poly(D,L-lactic-co-glycolic acid) meshes. Biomaterials 2006; 27: 5681-88

[12] Tamaki T, Akatsuka A, Ando K, et al. Identification of myogenicendothelial progenitor cells in the interstitial spaces of skeletal muscle. J Cell Biol 2002; 157: 571-77.

[13] Tamaki T, Akatsuka A, Okada Y, et al. Growth and differentiation potential of main- and side-population cells derived from murine skeletal muscle. Exp Cell Res 2003; 291: 83-90.

[14] Tamaki T, Okada Y, Uchiyama Y, et al. Clonal multipotency of skeletal muscle-derived stem cells between mesodermal and ectodermal lineage. Stem Cells 2007; 25: 2283-90.

[15] Okabe M, Ikawa M, Kominami K, et al. 'Green mice' as a source of ubiquitous green cells. FEBS Lett 1997; 407: 313-19.

[16] Tamaki T, Uchiyama Y, Okada Y, et al. Functional recovery of damaged skeletal muscle through synchronized vasculogenesis, myogenesis, and neurogenesis by muscle-derived stem cells. Circulation 2005; 112: 2857-66.

[17] Tamaki T, Okada Y, Uchiyama Y, et al. Synchronized reconstitution of muscle fibers, peripheral nerves and blood vessels by murine skeletal muscle-derived CD34(-)/45 (-) cells. Histochem Cell Biol 2007; 128: 349-60.

[18] Tamaki T, Akatsuka A, Okada Y, et al. Cardiomyocyte formation by skeletal muscle-derived multi-myogenic stem cells after transplantation into infarcted myocardium. PLoS One 2008; 3: e1789.

[19] Pepper MS, Ferrara N, Orci L, et al. Potent synergism between vascular endothelial growth factor and basic fibroblast growth factor in the induction of angiogenesis in vitro. Biochem Biophys Res Commun 1992; 189: 824-31.

[20] Vailhe B, Vittet D, Feige J-J. In vitro models of vasculogenesis and angiogenesis. Lab Invest 2001; 81: 439-52.

[21] Lange S, Altmann S, Brandt B, et al. Investigation of immunological approaches to enhance engraftment in a 1 Gy TBI canine hematopoietic stem cell transplantation model. Exp Hematol 2009; 37: 143-50. 
[22] Eggenhofer E, Popp FC, Renner P, et al. Allogeneic bone marrow transplantation restores liver function in Fah-knockout mice. Exp Hematol 2008; 36: 1507-13.

[23] Fu Y, Wang S, Liu Y, et al. Study on neural stem cell transplantation into natural rat cochlea via round window. Am J Otolaryngol 2009; 30: 8-16.

[24] Best TM, Shehadeh SE, Leverson G, et al. Analysis of changes in mRNA levels of myoblast- and fibroblast-derived gene products in healing skeletal muscle using quantitative reverse transcription-polymerase chain reaction. J Orthop Res 2001; 19: 565-72.

[25] Qin RF, Mao TQ, Gu XM, et al. Regulation of skeletal muscle differentiation in fibroblasts by exogenous MyoD gene in vitro and in vivo. Mol Cell Biochem 2007; 302: 233-39.

(C) Tono-Okada et al.; Licensee Bentham Open.

This is an open access article licensed under the terms of the Creative Commons Attribution Non-Commercial License (http://creativecommons.org/ licenses/by-nc/3.0/), which permits unrestricted, non-commercial use, distribution and reproduction in any medium, provided the work is properly cited. 